\title{
Biofilm production by Haemophilus influenzae and Streptococcus pneumoniae isolated from the nasopharynx of children with acute otitis media
}

Quentin Vermee ${ }^{1}$, Robert Cohen ${ }^{2,3,4}$, Constantin Hays ${ }^{1}$, Emmanuelle Varon ${ }^{5}$, Stephane Bonacorsi ${ }^{6}$, Stephane Bechet ${ }^{2}$, Franck Thollot ${ }^{7}$, François Corrard ${ }^{2}$, Claire Poyart ${ }^{1}$, Corinne Levy ${ }^{2,3}$ and Josette Raymond ${ }^{*}$

\begin{abstract}
Background: Biofilm production by Haemophilus influenzae and Streptococcus pneumoniae has been implicated in the pathogenesis of otitis media, mainly in chronic and recurrent cases. We studied the "in vitro" biofilm production by these 2 species isolated alone or together from the nasopharynx of children with acute otitis media.

Methods: The studied strains were from 3 pneumococcal conjugate vaccine (PCV) periods: pre-PCV7, post-PCV7/ pre-PCV13 and post-PCV13. A modified microtiter plate assay with crystal violet stain was used to study the biofilm production of $182 \mathrm{H}$. influenzae and 191 S. pneumoniae strains.

Results: Overall, 117/181 (64.6\%) H. influenzae and 128/191 (66.8\%) S. pneumoniae strains produced biofilm. The proportion of biofilm-producing $\mathrm{H}$. influenzae strains was greater with than without the isolation of S. pneumoniae in the same sample $(75.5 \%$ vs $52.3 \%, p=0.001)$. Conversely, the proportion of biofilm-producing S. pneumoniae strains was not affected by the presence or not of $\mathrm{H}$. influenzae (66.3\% vs $67.4 \%)$. S. pneumoniae serotypes $6 \mathrm{~B}$, 15B/C, 19A, 35F and 35B were the better biofilm producers (80\%). Serotypes 11 A, 14, 15A, 19F and 19A were more associated with $\mathrm{H}$. influenzae biofilm-producing strains. Overall, 89/94 (94.6\%) of cases with combined isolation showed biofilm production by S. pneumoniae or H. influenzae.

Conclusion: This study emphasizes the high proportion of biofilm production by $\mathrm{H}$. influenzae and S. pneumoniae strains isolated from the nasopharynx of children with acute otitis media, which reinforces the results of studies suggesting the importance of biofilm in the pathogenesis of acute otitis media.
\end{abstract}

Keywords: Haemophilus influenzae, Streptococcus pneumoniae, Biofilm, AOM, Naspharynx

\section{Background}

Acute otitis media (AOM) is the leading cause of bacterial infections in childhood, about 700 million cases each year, and the leading cause of antibiotic prescription [1]. More than $80 \%$ of children have at least one episode of AOM before age 4 years and $40 \%$ will have 6 or more recurrences by age 7 years $[2,3]$. Otitis media is a multifactorial disease, from uncomplicated AOM to more complex recurrent and chronic cases. Most cases of AOM resolve

\footnotetext{
* Correspondence: josette.raymond@aphp.fr

${ }^{1}$ Bactériologie, Hôpital Cochin, Université Paris Descartes, 27 rue du Faubourg Saint Jacques, 75679 Paris Cedex 14, France

Full list of author information is available at the end of the article
}

spontaneously, but complications that can occur include suppurative ones and long-term effects such as hearing loss.

The major pathogens involved in AOM, also called "otopathogens", are Streptococcus pneumoniae and non-typable Haemophilus influenzae (NT-Hi) (Casey et al., 2004, Block et al., 2004) as well as Moraxella catarrhalis $[4,5]$. NT- $H i$ is frequently associated with AOM treatment failure, recurrence and otitis media with effusion $[6,7]$.

The role of biofilm production has been suggested in several types of AOM. A biofilm is dynamic multimicrobial community adhering to a surface and enclosed in a 
matrix rich in exopolysaccharides, proteins, and nucleic acids. Bacteria living inside this structure are protected against external aggression such as the host immune system and antibiotic treatment [8]. In vivo biofilm production may involve multiple bacteria species, and several studies involving dual-species biofilm experiments have suggested complex inter-species interactions [9-11]. Some studies suggest that the persistence of bacterial species and notably NT- $\mathrm{Hi}$ in a biofilm-structured community plays a role in the pathogenesis of chronic, recurrent or non-responsive otitis media. Indeed, because of inefficient clearance of bacteria from the middle ear, the biofilm acts as a pathogen reservoir [12-16]. In situ hybridization or immunohistology techniques are probably the best methods to detect biofilm in these cases.

In the opposite spectrum of OM diseases, oto-pathogens are present in nasopharyngeal (NP) flora in biofilm, and some authors suggest that AOM episodes occur when bacteria escape from the biofilm surface to the surrounding space, in the region of adenoids, then Eustachian tube dysfunction promotes the penetration of the strains in the middle ear [17]. In these cases, hybridization and/or immunohistology techniques cannot be used, and other methods, animal models or in vitro methods, are needed to assess the role of biofilm. The capacity for NT-Hi and S. pneumoniae to produce biofilm can be demonstrated in vitro or in vivo. In vivo studies require a Chinchilla model of otitis media or direct detection by confocal laser scanning microscopy [18-22]. In vitro studies, such as microtiter plates or continuous-culture once-through flow cell, were described in several studies [14, 23-25]. Methods based on PCR techniques have been described, but they cannot discriminate between planktonic and biofilm-growing bacteria [26]. In our previous study, by using a modification of the microtiter plate assay with crystal violet (CV) stain, we found that $49 \%$ of $H$. influenzae strains isolated from children's nasopharynx produced biofilm [27].

Because of the pain caused by tympanocentesis, most clinical-practice guidelines for AOM generally do not recommend taking bacteriological samples of middle ear fluid except for treatment-failure and recalcitrant cases [28]. Kaur et al. used multilocus sequence typing to compare strains isolated from NP and middle-ear fluid samples in 34 children during an AOM episode and found the same sequence type of NT- $\mathrm{Hi}$ in $31 / 34$ children (84\%), which highlighted the similarity of strains isolated from both sites [28]. NP colonization with potential middle-ear pathogens is considered the initial event leading to AOM in humans, frequently preceded by or associated with viral infection $[29,30]$. Furthermore, some studies suggested that biofilm production ability differs by pneumococcal serotype $[24,25,31,32]$.
Here, we evaluated biofilm production by NT-Hi and $S$. pneumoniae strains isolated alone or together in the nasopharynx of the same patient and analyze biofilm production according to serotypes.

\section{Patients and methods}

\section{Patients}

NT-Hi and S. pneumoniae strains were isolated from NP samples from children with a diagnosis of AOM according to the Paradise criteria i.e. fever with or without otalgia and associated with bulging tympanic membrane and inflammation signs [33]. Because tympanocentesis is not a recommended first-line method for diagnosis of AOM, we used NP samples from patients with AOM and stringent criteria for the diagnosis of otitis. Bacterial strains were isolated from children included in the following 2 studies that used the same inclusion criteria.

Before PCV7 implementation, we performed 4 randomized trials of antibiotic treatment for AOM. These studies used standardized protocols: the same inclusion criteria for AOM, same age of enrolled children and bacteriological NP samples at enrolment to assess the NP oto-pathogen carriage [34]. These studies included 1807 children. After PCV implementation (PCV7 then PCV13), we investigated otopathogen carriage in 4405 children with AOM from 2001 to 2014 [35]. In both studies, the following data were collected: sex, age, history of AOM, antibiotic treatment during the previous 3 months, daycare attendance modality, vaccination status, and clinical symptoms (temperature $>38.5^{\circ} \mathrm{C}$, otalgia and/or conjunctivitis). Finally, 1779 children came from unpublished studies with the same design.

\section{Establishment of patient groups}

To study biofilm production, we randomly selected, for each PCV period, $182 \mathrm{NT}-H i$ strains (64 in pre-PCV7, 56 in post-PCV7/pre-PCV13, 61 in post-PCV13; 1 strain not evaluated) and 191 S. pneumoniae strains (64 in pre-PCV7, 64 in post-PCV7/pre-PCV13, 63 in post-PCV13) isolated from 277 of the 9798 children included in the 2 studies. For each PCV period, we defined 4 groups: NT-Hi strains isolated alone ( $n=86$; group 1$)$ or with $S$. pneumoniae $(n=96$; group 2), and S. pneumoniae strains isolated alone $(n=95$; group 3) or with NT- $H i(\mathrm{n}=96$; group 4$)$. Each group included 24 to 32 strains from the 3 periods. In the groups 1 and 2, we looked for the production of biofilm by $H$. influenzae and in the groups 2 and 4, we looked for the production of biofilm by $S$. pneumoniae.

\section{Ethical consent}

The protocols were approved by the Saint Germain en Laye Hospital Ethics Committee. Written informed consent was obtained from parents or legal representatives of children. 


\section{Methods}

Samples were taken as previously described [34, 35] stored at ambient temperature and arrived within $48 \mathrm{~h}$ at the Centre National de Référence des Pneumocoques (Hôpital Européen Georges Pompidou, Paris, France) and the Robert Debré Hospital Bacteriological Laboratory (Paris, France).

Culture was performed according to the same method as previously [34, 35]. Identification was confirmed by matrix-assisted laser desorption ionization-time of flight mass spectrometry (MALDI-TOF MS, Bruker Daltonics, Germany) for $H$. influenzae and MALDI-TOF MS and optochin susceptibility for S. pneumoniae.

S. pneumoniae serotyping was performed at the French National Reference Center for S. pneumoniae (CNRP, Hôpital Européen Georges Pompidou, Paris, France) [34, 35].

Antibiotic susceptibility of S. pneumoniae and H. influenzae was determined according to $t$ those proposed by the CASFM/EUCAST (www.sfm-microbiologie.org/ UserFiles/files/casfm/CASFM2016_V1_0_FEVRIER.pdf).

\section{Microtiter biofilm formation assay}

Biofilm production was evaluated by a modification of the microtiter plate assay with crystal violet $(\mathrm{CV})$ stain as described for NT-Hi [27]. This assay was based on the ability of bacteria to adhere to solid polystyrene surfaces by using biofilm. The biofilm production assay was adapted for S. pneumoniae. Briefly, S. pneumoniae isolates were sub-cultured on $5 \%$ blood agar (Biomérieux, La Balme Les Grottes, France) and incubated at $37{ }^{\circ} \mathrm{C}$ for $24 \mathrm{~h}$ under $5 \% \mathrm{CO}_{2}$. Then, $\mathrm{S}$. pneumoniae isolates were grown for $8 \mathrm{~h}$ at $35^{\circ} \mathrm{C}$ with $5 \% \mathrm{CO}_{2}$ in $\mathrm{BHI}$ (Becton Dickinson, Le Pont-De-Claix, France). Thereafter, the protocol was identical to that for NT-Hi. For each strain, the assay was performed in triplicate and repeat in 2 independent experiments.

A Staphylococcus aureus strain known as a strong biofilm producer was used as a positive control in each experiment and a Streptococcus agalactiae strain known as a poor biofilm producer was used as a negative control. These strains were kindly provided by the Streptococci national reference center (Pr C. Poyart, Cochin Hospital, Paris, France). For each assay, the BHI was tested alone to calculate the biofilm formation index (BFI).

\section{Determination of cutoff values}

The biofilm formation index (BFI) was determined by applying 3 different formulas. The first was derived from Kadurugamuwa et al. [36]. The authors calculated the $\mathrm{BFI}$ at optical density $595 \mathrm{~nm}$ as $\mathrm{BFI}=\mathrm{AB}-\mathrm{CW}$, where $\mathrm{AB}$ represents stained wells containing attached bacteria and CW, stained control wells containing bacteria-free medium only, here supplemented with BHI. The second method, described by Soto et al. [37], used the same index with a different formula: $\mathrm{BFI}=\mathrm{AB} / \mathrm{CW}$. The third method was according to Niu et al. (2004): BFI $=(A B-$ $\mathrm{CW} / \mathrm{G}$, where $\mathrm{G}$ is bacterial planktonic growth control [38]. The assays were performed in triplicate. Finally, the results for each method were studied by terciles to classify the biofilm production semi-quantitatively into 3 categories: strong production (S), moderate production (M) and absence of production (negative; N) according to the cutoff values proposed by Naves et al. [39]. However, unlike Naves et al., we grouped the strains with moderate or low biofilm production [39].

Naves et al. showed that biofilm production is strongly altered by culture conditions, environmental factors and methodology. For these reasons and to avoid these pitfalls, we combined the 3 different BFI calculation methods. This method of calculation was validated to study the biofilm production by $H$. influenzae and published in 2014 [27].

\section{Statistical analysis}

Double data entry involved use of the software 4D v12.5. Statistical analysis involved use of Stata SE 13.1 (Stata Corp., College Station, TX, USA). Pearson chi-square test or Fisher exact test was used to compare groups (two-tailed tests). Potential risk factors were identified by univariate analysis $(p<0.25)$ and introduced in multivariate logistic regression models. Only significant risk factors $(p<0.05)$ remained in the final model. For statistical analysis, we merged strong and moderate biofilm-producing bacteria to compare 2 groups: "moderate or strong biofilm-producer" versus "low biofilm-producer" for both NT-Hi and S. pneumoniae. $P<0.05$ was considered statistically significant.

\section{Results}

\section{Methods for calculating the biofilm production}

The cut-offs used to determine the intensity of biofilm production in isolates of $S$. pneumoniae and $H$. influen$z a e$ were as followed where $\mathrm{AB}$ represented the stained wells containing attached bacteria, CW the stained control wells containing bacteria-free medium only and $G$ the bacterial growth control i) AB - CW: strong production $>0.30$, moderate production $0.10-0.30$, negative < 0.10 ii) $\mathrm{AB} / \mathrm{CW}$ : strong production $>6$, moderate production $2-6$, negative $<2$ iii $)[(A B-C W) / G]$ : strong production $>1.10$, moderate production $0.35-1.10$, negative $<0.35$.

Strains were classified according to agreement of at least 2 of the 3 methods used to calculate biofilm production by S. pneumoniae (Additional file 1: Supplementary Data S1) and $H$. influenzae (Additional file 2: Supplementary Data S2). Among 182 NT-Hi strains, 62 (34\%) were classified as strong biofilm producers, 55 (30.2\%) moderate producers and 64 (35.1\%) non-producers. One strain could 
not be evaluated. Overall, 117/181 (64.6\%) NT- $H i$ strains produced biofilm (Table 1). By using the same classification, among the 191 S. pneumoniae strains, 63 (33\%) were classified as strong biofilm producers, 64 (33.5\%) moderate producers and 63 (33\%) non-producers. Overall, 128/ 191 (66.8\%) S. pneumoniae strains produced biofilm (Table 1).

\section{Evolution of the proportion of biofilm-producing strains} Between the pre-PCV7 and post-PCV7/pre-PCV13 periods, the proportion of NT- $H i$ biofilm-producing strains was stable (67.2 and $76.8 \%$, respectively, $\mathrm{p}=\mathrm{ns}$ ), whether isolated alone or with S. pneumoniae strains (Table 2). However, the proportion of NT-Hi biofilm-producing strains was lower in the post-PCV13 than post-PCV7/ pre-PCV13 period for strains isolated alone (62.5 and $36.7 \%$, respectively, $p=0.05$ ) or with $S$. pneumoniae strains ( 87.5 and $63.3 \%$, respectively, $p=0.02)$. Overall, the proportion of NT-Hi biofilm-producing strains was greater when isolated with S. pneumoniae $(75.5 \%$ vs $52.3 \%, p=0.001$ ) (Table 2).

Conversely, the proportion of S. pneumoniae biofilmproducing strains did not change over the study periods, whether isolated alone (65.6 and $67.7 \%$ in pre-PCV7 and post-PCV13 periods) or with NT-Hi strains (71.9 and $62.5 \%$ in pre-PCV7 and post-PCV13 periods). Overall, the proportion of $S$. pneumoniae biofilm-producing strains did not differ whether isolated alone or with NT-Hi strains [67.4\% vs. 66.3\%, $p=0.92]$ (Table 2).

\section{Characteristics of S. pneumoniae biofilm-producing strains}

We found no differences in biofilm-producing ability by pneumococcal serotype (Table 2). The proportion of $S$. pneumoniae serotypes included in PCV7 (54/191) that produced biofilm was similar to that for other serotypes: $57.4 \%(31 / 54)$ versus $70.6 \%$ (96/137), $p=0.09$ (data not shown). Moreover, the proportion did not differ between the 6 additional $S$. pneumoniae serotypes included in PCV13 and other serotypes [78.5\% (22/28) vs $64.4 \%$ (105/ 163), $p=0.14)]$. Strains of serotypes $6 \mathrm{~B}, 15 \mathrm{~B} / \mathrm{C}, 19 \mathrm{~A}, 35 \mathrm{~F}$, and $35 \mathrm{~B}$ produced biofilm in more than $80 \%$ of the cases. In contrast, strains of serotypes 23B, 23F, $19 \mathrm{~F}$ were the lowest producers ( $40 \%$ of strains) (Table 2 ).

The $S$. pneumoniae serotypes frequently isolated with NT- $H i$ strains were $11 \mathrm{~A}, 14,15 \mathrm{~A}, 15 \mathrm{~B} / \mathrm{C}, 19 \mathrm{~A}, 19 \mathrm{~F}, 23 \mathrm{~F}$ and $23 \mathrm{~B}$, representing $60.4 \%$ (58/96) of the combination. $H$. influenzae biofilm-producing strains were isolated more often with serotypes 11A, 14, 15A, 19F and 19A. This association did not affect biofilm production by $S$. pneumoniae.

Susceptibility or resistance to penicillin did not differ with and without biofilm production: $64.2 \%$ (61/96) versus $69.5 \%$ (66/95), $p=0.44$.

Overall, $75.5 \%$ (72/96) of NT- $H i$ strains produced biofilm when isolated with S. pneumoniae strains. Serotypes of $S$. pneumoniae were not associated with biofilm production by NT-Hi strains.

When isolated from the same nasopharynx, S. pneumoniae or $H$. influenzae or both produced biofilm in 94.6\% (89/94) of cases (Table 3).

\section{Relation between clinical signs and biofilm production}

Table 3 presents the demographic characteristics and clinical signs by biofilm production. Biofilm was produced significantly more often by NT-Hi strains isolated from children with than without fever (temperature $>38.5^{\circ} \mathrm{C}$ ) or fever + otalgia $[75 \%(42 / 56)$ vs $59.7 \%(74 / 124), p=$ 0.047 and $74.6 \%(50 / 67)$ vs $56.5 \%(65 / 112), p=0.025]$. These relations were not observed for S. pneumoniae ( $p=$ 0.46 and $p=0.87$, respectively).

Antimicrobial susceptibility and serotyping of the strains The proportion of resistant strains significantly decreased from the pre-PCV7 to post-PCV13 periods for both NT-Hi and S. pneumoniae. Among NT-Hi strains, the

Table 1 Biofilm production by H. influenzae and S. pneumoniae isolated alone or together by pneumococcal conjugate vaccine (PCV) period

\begin{tabular}{lllll}
\hline Biofilm production & Pre-PCV7 & Post-PCV7/Pre-PCV13 & Post-PCV13 & Total \\
\hline H. influenzae & & & & \\
$\quad$ Isolated alone & $19 / 32(59.4 \%)$ & $15 / 24(62.5 \%)^{\text {a }}$ & $11 / 30(36.7 \%)^{\text {a }}$ & $45 / 86(52.3 \%)^{c}$ \\
Isolated with S. pneumoniae & $24 / 32(75 \%)$ & $28 / 32(87.5 \%)^{\mathrm{b}}$ & $20 / 32(63.3 \%)^{\mathrm{b}}$ & $72 / 96(75.5 \%)^{\mathrm{c}}$ \\
$\quad$ Total & $43 / 64(67.2 \%)$ & $43 / 56(76.8 \%)$ & $31 / 61(50.8 \%)$ & $117 / 181(64.6 \%)$ \\
S. pneumoniae & & & & \\
$\quad$ Isolated alone & $21 / 32(65.6 \%)$ & $22 / 32(68.8 \%)$ & $21 / 31(67.7 \%)$ & $64 / 95(67.4 \%)$ \\
Isolated with H. influenzae & $23 / 32(71.9 \%)$ & $21 / 32(64.5 \%)$ & $20 / 32(62.5 \%)$ & $64 / 96(66.3 \%)$ \\
$\quad$ Total & $44 / 64(68.8 \%)$ & $43 / 64(66.7 \%)$ & $41 / 63(65.1 \%)$ & $128 / 191(66.8 \%)$ \\
\hline
\end{tabular}

${ }^{\mathrm{a}} \mathrm{H}$. influenzae isolated alone between the post-PCV7/prePCV13 and post-PCV13 periods $p=0.05$

${ }^{\mathrm{b}} \mathrm{H}$. influenzae associated with S. pneumoniae between the post-PCV7/pre-PCV13 and post-PCV13 periods $p=0.02$

c Total of $\mathrm{H}$. influenzae alone or associated with S. pneumoniae independent of period $p=0.001$ 


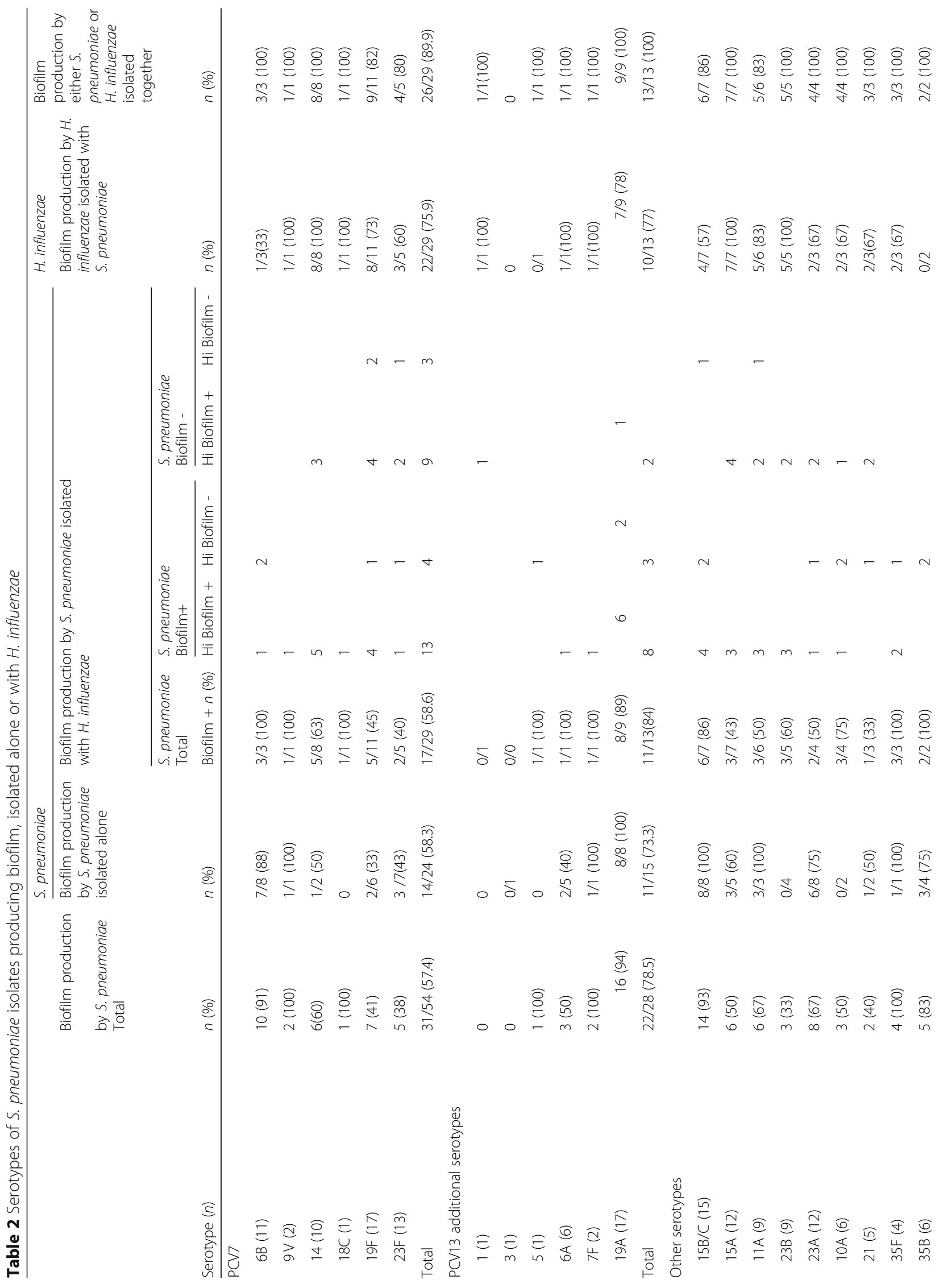




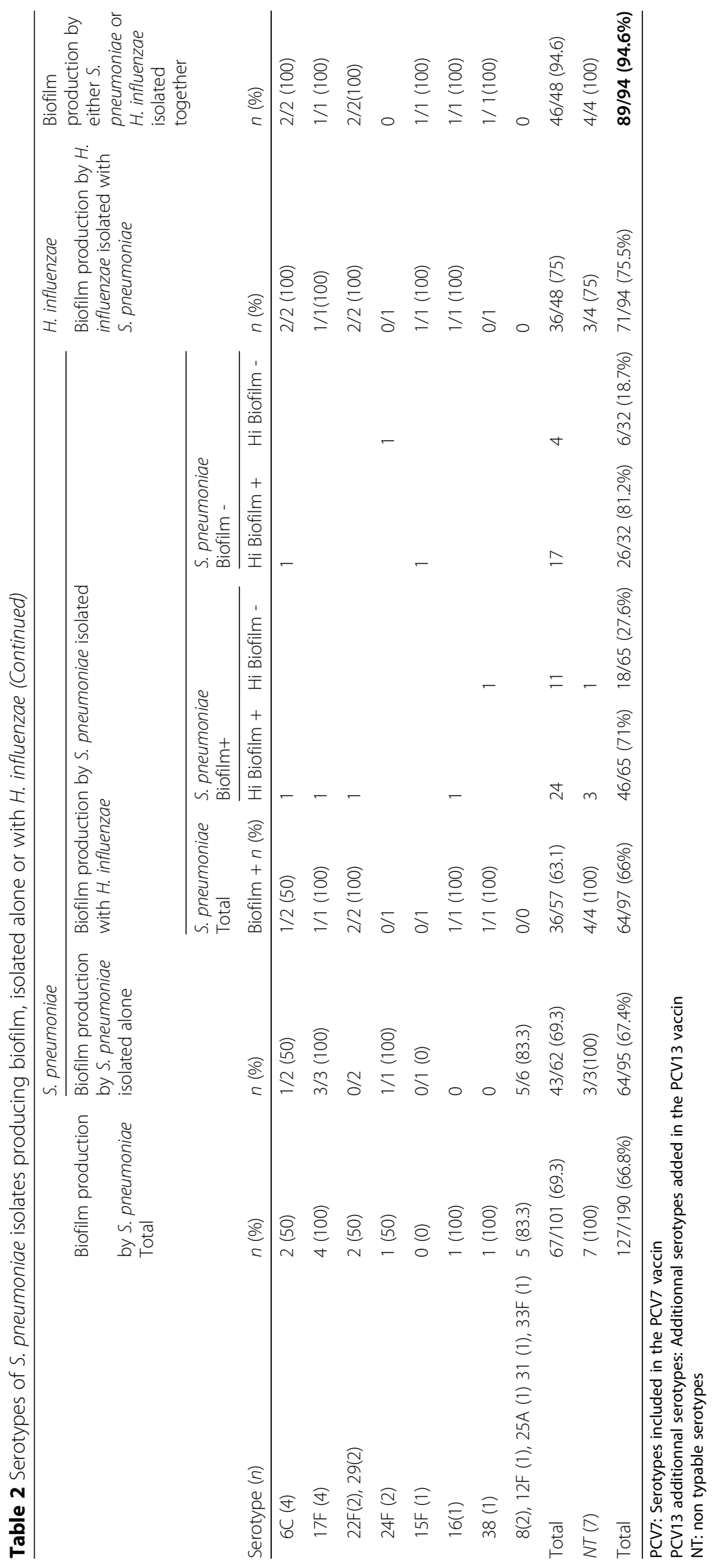


Table 3 Demographic characteristics and clinical signs of children according to the biofilm production

\begin{tabular}{lllll}
\hline Characteristics and clinical signs & Biofilm production by H. influenzae & $p^{*}$ & Biofilm production by S. pneumoniae & $p^{*}$ \\
\hline Day-care center & $47 / 72(65.3)$ & 0.66 & $42 / 63(66.7)$ & 0.99 \\
Recurrent acute otitis media & $22 / 33(66.7)$ & 0.77 & $19 / 29(65.5)$ & 0.87 \\
Conjunctivitis & $59 / 97(60.8)$ & 0.27 & $42 / 66(63.6)$ & 0.52 \\
Otalgia & $96 / 145(66.2)$ & 0.26 & $105 / 156(67.3)$ & 0.68 \\
Temperature $>38.5^{\circ} \mathrm{C}$ & $42 / 56(75.0)$ & $\mathbf{0 . 0 4 7}$ & $45 / 71(63.4)$ & 0.46 \\
Fever + otalgia & $50 / 67(74.6)$ & $\mathbf{0 . 0 2 5}$ & $58 / 86(67.4)$ & 0.87 \\
Antibiotics 3 months before enrollment & $53 / 87(60.9)$ & 0.34 & $55 / 82(67.1)$ & 0.95 \\
\hline
\end{tabular}

${ }^{*} p$ comparing biofilm strain producers and non-producers

proportion of $\beta$-lactamase-producing strains decreased from $51.5 \%(33 / 64)$ in the pre-PCV7 period to $17.8 \%$ $(10 / 56)$ and $17.7 \%(11 / 62)$ in the post-PCV7/prePCV13 and post-PCV13 periods, respectively $(p<$ $0.001)$. As well, the proportion of $S$. pneumoniae strains with decreased susceptibility to penicillin decreased from $65.7 \%$ in the pre-PCV7 period to $45.4 \%(29 / 64)$ and $39.7 \%(25 / 63)$ in the post-PCV7/pre-PCV13 and post-PCV13 periods $(p=0.003)$. Among S. pneumoniae strains, the proportion of those with erythromycin susceptibility increased from $46.8 \%(30 / 64)$ to $81 \%(51 / 63)$ between the post-PCV7/pre-PCV13 and post-PCV13 periods, respectively $(\mathrm{p}<0.001)$.

After PCV implementation, the distribution of the S. pneumoniae serotypes changed according to the study period (Additional file 3: Supplementary Data S3). In the pre-PCV7 period, the serotypes of $47 / 64$ S. pneumoniae strains were included in PCV7, 10/64 involved the 6 additional serotypes included in PCV13, and 3 were serotype $15 \mathrm{~B} / \mathrm{C}$. In the post-PCV7/pre-PCV13 period, the serotypes of $6 / 64 \mathrm{~S}$. pneumoniae strains were included in PCV7, 19/64 involved the 6 additional serotypes included in PCV13, and 38/62 were of various serotypes. In the post-PCV13 period, only 1/63 serotypes was included in PCV13.

\section{Discussion}

To our knowledge, it is the first study exploring in a large cohort the production of biofilms of $H$. influenzae and S. pneumoniae isolated alone or together from NP flora of children with AOM. Previously, the role of biofilm production in AOM was mainly explored for $S$. pneumoniae $[25,31,40]$ or $H$. influenzae $[22,27,41]$ or both in Chinchilla models [42].

We used here three methods of calculation because there is no reference method for biofilm production. We found high biofilm production for both $S$. pneumoniae and $H$. influenzae (66.8 and $64.4 \%$, respectively) with no variation in proportion for $S$. pneumoniae over PCV implementation periods. Of note, the proportion of NT-Hi biofilm-producing strains was greater when $H$. influenzae strains were isolated with $S$. pneumoniae, which agreed with the results of Hong et al. [10].

When S. pneumoniae and $H$. influenzae were isolated together, $93.7 \%$ of cases showed biofilm production by S. pneumoniae and/or $H$. influenzae. Residence of a bacterium within a biofilm allows for global changes in gene and protein expression profiles, which has many effects on cell physiology, promoting adhesion and cohesion properties of biofilm cells, thereby increasing its persistence [43]. Recent studies demonstrated that $H$. influenzae and $S$. pneumoniae modulate the expression of each other's virulence genes, which results in persistent biofilm, mainly by upregulati type IV pilus structural protein (pilA) by $H$. influenzae, thereby playing an important role in adhesion and biofilm stability $[9,12]$. Hong et al. reported significantly downregulated expression of pneumococcal genes regulating autolysis and fratricide, lyt $A$ and $c b p D$, on co-culture with $\mathrm{NT}-\mathrm{Hi}$, which suggests that pneumococcal survival and biofilm production can be enhanced in the presence of NT-Hi [10] .

We found that S. pneumoniae serotypes $6 \mathrm{~B}, 15 \mathrm{~B} / \mathrm{C}$, $19 \mathrm{~A}, 35 \mathrm{~F}$ and $35 \mathrm{~B}$ were the best biofilm producers. Previously, serotypes $14,6 \mathrm{~B}, 15 \mathrm{~B} / \mathrm{C}$, and $11 \mathrm{~A}$ were found efficient in producing biofilm $[25,40]$, whereas Domenech et al., reported serotypes $35 \mathrm{~B}$ and $11 \mathrm{~A}, 19 \mathrm{~A}$ as efficient [24]. Therefore, these results support the validity of our method because the 5 serotypes we found as the best biofilm producers were those previously described. Analysis of a large database of AOM serotypes revealed that serotype 19A has the highest disease potential for AOM [44]. The high production of biofilm may be one explanation for this phenomenon.

Our study has several limitations. The first is the lack of direct evaluation of biofilm in biological samples in middle ear fluid (MEF) with alternative methods such as confocal microscopy after live/dead staining [45, 46]. However, we assumed that this method could not be used for a large population with AOM. Second, one can argue that the studied strains were isolated from the 
nasopharynx and not from MEF. We postulate, as do other authors, that the reservoir of bacterial species implicated in AOM is the nasopharynx, and their carriage precedes AOM [29, 30]. Previously we demonstrated no significant difference in biofilm production between NT- $H i$ strains from MEF and NP samples [27]. In a previous prospective study, Bingen et al., using pulsed-field gel electrophoresis in conjunctivitis-otitis syndrome, revealed identical NT-Hi strains isolated from MEF and conjunctivitis tissue [47] . Van Dongen et al., in a systematic review found NT-Hi strains isolated from both MEF and NP samples in $80 \%$ of cases [48]. More recently, Van Hoecke et al., investigating the presence of otopathogenic bacteria in middle ear effusion and adenoids of children with chronic otitis, found NT-Hi and S. pneumoniae, isolated from both locations, genetically identical in 13/14 cases [22]. The third limitation is that we could have used knock-out mutants as a control. However, many genes appear to be involved, although the role of each appears to vary when biofilm is produced in batch or continuous culture. Proteomic studies have revealed an increase in number of proteins synthesized de novo and differences in protein production patterns during S. pneumoniae biofilm development [23]. In these conditions, in vivo studies are difficult. Another limitation is that these isolates came only from children with $\mathrm{AOM}$ and not from healthy children or children with chronic AOM. However, these results support the hypothesis that multispecies biofilm is the basis for the chronicity of otitis media as previously suggested [13]. Finally, even if a larger number of strains would have allowed us to have more statistical power, our sample allowed us to detect some statistical differences.

The last limitation of our study is that biofilm production was studied in monoculture because of the different culture requirements of the 2 strains. One reason was the difficulty in co-cultivating the 2 bacteria because of the rapid lysis of $S$. pneumoniae in a liquid medium. The incubation time required for the experiment was not the same for both bacteria.

The role of biofilm in otitis media is not fully understood, and we lack a universally accepted or feasible method to study biofilm formation in vitro and in vivo in humans. We are aware that we are simply bringing some modest information to a complex puzzle. However, if our study has several limitations, it has also several strengths as follows. 1) To our knowledge, it is the first study exploring in a large cohort the production of biofilm of $H$. influenzae and S. pneumoniae isolated from NP flora of children with AOM. 2) We were able to demonstrate $\mathrm{H}$. influenzae and/or S. pneumoniae biofilm production in all the clinical situations and more particularly by NT- $H i$ strains isolated from children with fever or fever associated with otalgia; this seems an important point reinforcing biofilm production as a ubiquitous phenomenon in carriage. 3) More than $60 \%$ of S. pneumoniae and $H$. influenzae strains produced biofilm, and this proportion increased significantly for $H$. influenzae when the 2 bacterial species were isolated in the same sample. Furthermore, biofilm production globally did not differ by period or vaccine and non-vaccine type. 4) Our results agree with those of previous studies regarding the ability of some S. pneumoniae serotypes to produce biofilm.

\section{Conclusion}

This study highlighted the biofilm production among the 2 main oto-pathogens strains $(93.7 \%$ of cases showed biofilm production by S. pneumoniae or $H$. influenzae). The proportion of NT-Hi biofilm-producing strains was greater when isolated with S. pneumoniae. Furthermore, some $S$. pneumoniae serotypes produced more biofilm than did other.

\section{Additional files}

\begin{abstract}
Additional file 1: Supplementary Data S1: Different cut-offs used to determine the biofilm production by S. pneumoniae: the different cut-offs used were as followed where $A B$ represented the stained wells containing attached bacteria, CW the stained control wells containing bacteriafree medium only and $\mathrm{G}$ the bacterial growth control i) $\mathrm{AB}-\mathrm{CW}$ : strong production $>0.30$, moderate production 0.10-0.30, negative $<0.10$ ii) $A B /$ CW: strong production $>6$, moderate production $2-6$, negative $<2$ iii) $[(A B-$ CW) / G]: strong production $>1.10$, moderate production $0.35-1.10$, negative $<0.35$. Strains were classified according to agreement of at least 2 of the 3 methods used to calculate biofilm production. (XLS $195 \mathrm{~kb}$ )

Additional file 2: Supplementary Data S2: Different cut-offs used to determine the biofilm production by $H$. influenzae: the different cut-offs used were as followed where $A B$ represented the stained wells containing attached bacteria, CW the stained control wells containing bacteria-free medium only and $G$ the bacterial growth control i) $A B-C W$ : strong production $>0.30$, moderate production $0.10-0.30$, negative $<0.10$ ii) $A B / C W$ : strong production $>6$, moderate production $2-6$, negative $<2$ iii) $[(A B-C W)$ / G]: strong production $>1.10$, moderate production $0.35-1.10$, negative $<$ 0.35. Strains were classified according to agreement of at least 2 of the 3 methods used to calculate biofilm production. (XLSX $76 \mathrm{~kb}$ )
\end{abstract}

Additional file 3: Supplementary Data S3: Distribution of S. pneumoniae serotypes by study period (pre-PCV7, post-PCV7/pre-PCV13 and postPCV13). (DOCX $15 \mathrm{~kb}$ )

\section{Abbreviations}

AOM: Acute otitis media; BFI: Biofilm formation index; BHI: Brain heart infusion; CASFM/EUCAST: Comité de l'Antibiogramme de la Société Française de Microbiologie/European Committee on Antimicrobial Susceptibility Testing; CNRP: Centre National de Référence des Pneumocoques; CV: Crystal violet; MALDI-TOF MS: Matrix-assisted laser desorption ionization-time of flight mass spectrometry; MEF: Middle ear fluid; NP: Nasopharyngeal; NTHi: Haemophilus influenzae; PCR: Polymerase chain reaction; PCV: Pneumococcal conjugate Vaccine

\section{Acknowledgements}

We are grateful to the following paediatricians from the Association Clinique et Thérapeutique Infantile du Val de Marne (ACTIV) who provided the samples: E. Sobaral, M. Boucherat, I. Ramay, M. Fernandes, C. Prieur, D. Kern and A. Prieur. 


\section{Funding}

This work was supported in part by Pfizer and by Association Clinique et Thérapeutique Infantile du Val de Marne (ACTIV).

The sponsor had no role in data collection, data analysis, data interpretation, writing of the report or decision to publish.

\section{Availability of data and materials}

The datasets used and/or analyzed during the current study are available from the corresponding author on reasonable request.

\section{Authors' contributions}

QR performed experiments and wrote the manuscript. JR headed the study and wrote the manuscript. $\mathrm{CH}$ performed experiments and wrote the manuscript. RC designed the study and wrote the manuscript. SB1 and EV provided the strains. SB2 and CL managed data and performed statistical analysis. FT and FC collected the samples from children. All authors read and approved the final manuscript.

\section{Ethics approval and consent to participate}

The protocols were approved by the Saint Germain en Laye Hospital Ethics Committee. Written informed consent was obtained from parents or legal representatives of children for use of samples and data.

\section{Competing interests}

The authors declare that they have no competing interests.

\section{Publisher's Note}

Springer Nature remains neutral with regard to jurisdictional claims in published maps and institutional affiliations.

\section{Author details}

${ }^{1}$ Bactériologie, Hôpital Cochin, Université Paris Descartes, 27 rue du Faubourg Saint Jacques, 75679 Paris Cedex 14, France. ${ }^{2}$ Association Clinique et Thérapeutique Infantile du Val de Marne (ACTIV), 94 Saint-Maur des Fossés, France. ${ }^{3}$ IMRB- GRC GEMINI, Clinical Research Center (CRC), Centre Hospitalier Intercommunal de Créteil, Université Paris Est, Créteil, France. ${ }^{4}$ Service de Néonatologie, Centre Hospitalier Intercommunal de Créteil, Créteil, France. ${ }^{5}$ Bactériologie, Hôpital Georges Pompidou, Université Paris Descartes, Paris, France. ${ }^{6}$ Bactériologie, Hôpital Robert Debré, Université Diderot, Paris, France. ${ }^{7}$ Essey les Nancy, AFPA, Paris, France.

Received: 12 April 2018 Accepted: 26 December 2018

Published online: 11 January 2019

\section{References}

1. Monasta L, Ronfani L, Marchetti F, Montico M, Vecchi Brumatti L, Bavcar A, et al. Burden of disease caused by otitis media: systematic review and global estimates. PLoS One. 2012;7(4):e36226. https://doi.org/10.1371/ journal.pone.0036226 Epub 2012 Apr 30.

2. Teele DW, Klein JO, Rosner B. Epidemiology of otitis media during the first seven years of life in children in greater Boston: a prospective, cohort study. J Infect Dis. 1989;160:83-94.

3. Vergison A, Dagan R, Arguedas A, Bonhoeffer J, Cohen R, Dhooge I, et al. Otitis media and its consequences: beyond the earache. Lancet Infect Dis. 2010;10(3):195-203. https://doi.org/10.1016/S1473-3099(10)70012-8.

4. Block SL, Hedrick J, Harrison CJ, Tyler R, Smith A, Findlay R, Keegan E. Community-wide vaccination with the heptavalent pneumococcal conjugate significantly alters the microbiology of acute otitis media. Pediatr Infect Dis J. 2004;23(9):829-33.

5. Casey JR, Pichichero ME. Changes in frequency and pathogens causing acute otitis media in 1995-2003. Pediatr Infect Dis J. 2004;23(9):824-8.

6. Barkai G, Leibovitz E, Givon-Lavi N, Dagan R. Potential contribution by nontypable Haemophilus influenzae in protracted and recurrent acute otitis media. Pediatr Infect Dis J. 2009;28(6):466-71.

7. Caeymaex L, Varon E, Levy C, Béchet S, Derkx V, Desvignes V, Doit C, Cohen R. Characteristics and outcomes of acute otitis media in children carrying Streptococcus pneumoniae or Haemophilus influenzae in their nasopharynx as a single otopathogen after introduction of the heptavalent pneumococcal conjugate vaccine. Pediatr Infect Dis J. 2014;33(5):533-6.

8. Costerton JW, Stewart PS, Greenberg EP. Bacterial biofilms: a common cause of persistent infections. Science. 1999;284(5418):1318-22.
9. Cope EK, Goldstein-Daruech N, Kofonow JM, Christensen L, McDermott B, Monroy $F$, et al. Regulation of virulence gene expression resulting from Streptococcus pneumoniae and nontypeable Haemophilus influenzae interactions in chronic disease. PLoS One. 2011;6(12):e28523. https://doi.org/ 10.1371/journal.pone.0028523 Epub 2011 Dec 5.

10. Hong W, Khampang P, Erbe C, Kumar S, Taylor SR, Kerschner JE. Nontypeable Haemophilus influenzae inhibits autolysis and fratricide of Streptococcus pneumoniae in vitro. Microbes Infect. 2014;16(3):203-13.

11. Tikhomirova A, Trappetti C, Paton JC, Kidd SP. The outcome of NT-Hi and S. pneumoniae inter-species interactions depends on $\mathrm{pH}$, nutrient availability and growth phase. Int J Med Microbiol. 2015;305:881-92.

12. Bakaletz LO. Bacteria biofilms in otitis media: evidence and relevance. Pediatr Infect Dis J. 2007:26:S17-9.

13. Dagan R, Pelton S, Bakaletz L, Cohen R. Prevention of early episodes of otitis media by pneumococcal vaccines might reduce progression to complex disease. Lancet Infect Dis. 2016;16:480-92.

14. Murphy TF, Kirkham C. Biofilm formation by nontypeable Haemophilus influenzae: strain variability, outer membrane antigen expression and role of pili. BMC Microbiol. 2002;2:7.

15. Nistico L, Kreft R, Gieseke A, Coticchia JM, Burrows A, Khampang P, et al. Adenoid reservoir for pathogenic biofilm bacteria. J Clin Microbiol. 2011 49(4):1411-20. https://doi.org/10.1128/JCM.00756-10 Epub 2011 Feb 9.

16. Wolcott RD, Ehrlich GD. Biofilms and chronic infections. JAMA. 2008;299: 2682-4.

17. Coticchia JM, Chen M, Sachdeva L, Mutchnick S. New paradigms in the pathogenesis of otitis media in children. Front Pediatr. 2013;23:1-52. https:// doi.org/10.3389/fped.2013.00052.

18. Hall-Stoodley L, Hu FZ, Gieseke A, Nistico L, Nguyen D, Hayes J, et al. Direct detection of bacterial biofilms on the middle-ear mucosa of children with chronic otitis media. JAMA. 2006;296(2):202-11.

19. Murrah KA, Pang B, Richardson S, Perez A, Reimche J, King L, et al. Nonencapsulated Streptococcus pneumoniae causes otitis media during single-species infection and during polymicrobial infection with nontypeable Haemophilus influenzae. Pathog Dis. 2015;73(5). https://doi.org/ 10.1093/femspd/ftu011 Epub 2014 Dec 4.

20. Reid SD, Hong W, Dew KE, Winn DR, Pang B, et al. Streptococcus pneumoniae forms surface-attached communities in the middle ear of experimentally infected chinchillas. J Infect Dis. 2009;199(6):786-94.

21. Thornton RB, Rigby PJ, Wiertsema SP, Filion P, Langlands J, Coates HL, et al. Multi-species bacterial biofilm and intracellular infection in otitis media. BMC Pediatr. 2011;24:11-94. https://doi.org/10.1186/1471-2431-11-94.

22. Van Hoecke H, De Paepe AS, Lambert E, Van Belleghem JD, Cools P, Van Simaey $L$, et al. Haemophilus influenzae biofilm formation in chronic otitis media with effusion. Eur Arch Otorhinolaryngol. 2016;273(11):3553-60.

23. Allegrucci M, Hu FZ, Shen K, Hayes J, Ehrlich GD, Post JC, Sauer K. Phenotypic characterization of Streptococcus pneumoniae biofilm development. J Bacteriol. 2006;188(7):2325-35.

24. Domenech M, Damián D, Ardanuy C, Liñares J, Fenoll A, García E. Emerging Non-PCV13 Serotypes 11A and 35B of Streptococcus pneumoniae Show High Potential for Biofilm Formation In Vitro. PLoS One. 2015;10(4):e0125636. https://doi.org/10.1371/journal.pone.0125636.

25. Tapiainen T, Kujala T, Kaijalainen T, Ikäheimo I, Saukkoriipi A, Renko M, et al. Biofilm formation by Streptococcus pneumoniae isolates from pediatrics patients. APMIS. 2010;118(4):255-60. https://doi.org/10.1111/j.1600-0463. 2010.02587.

26. Frank DN, Wilson SS, St Amand AL, Pace NR. Culture-independent microbiological analysis of Foley urinary catheter biofilms. PLoS One. 2009:4: e7811.

27. Mizrahi A, Cohen R, Varon E, Bonacorsi S, Bechet S, Poyart C, Levy C, Raymond J. Non typable-Haemophilus influenzae biofilm formation and acute otitis media. BMC Infect Dis. 2014;19:14-400. https://doi.org/10.1186/ 1471-2334-14-400

28. Kaur R, Chang A, Xu Q, Casey JR, Pichichero M. Phylogenetic relatedness and diversity of non-typable Haemophilus influenzae in the nasopharynx and middle ear fluid of children with acute otitis media. J Med Microbiol. 2011:60:1841-8.

29. Chonmaitree T, Trujillo R, Jennings $K$, Alvarez-Fernandez P, Patel JA, Loeffelholz MJ, Nokso Koivisto J, Matalon R, Pyles RB, Miller AL, McCormick DP. Acute Otitis Media and Other Complications of Viral Respiratory Infection. Pediatrics. 2016;137(4):e20153555. https://doi.org/10.1542/peds. 2015-3555. 
30. Faden H, Duffy L, Wasielewski R, Wolf J, Krystofik D, Tung Y. Relationship between nasopharyngeal colonization and the development of otitis media in children. Tonawanda/Williamsville Pediatrics. J Infect Dis. 1997;175(6): $1440-5$.

31. Domenech M, Araújo-Bazán L, García E, Moscoso M. In vitro biofilm formation by Streptococcus pneumoniae as a predictor of post-vaccination emerging serotypes colonizing the human nasopharynx. Environ Microbiol. 2014;16:1193-201.

32. Moscoso M, García E, López R. Biofilm formation by Streptococcus pneumoniae: role of choline, extracellular DNA, and capsular polysaccharide in microbial accretion. J Bacteriol. 2006;188:7785-95.

33. Paradise JL. Treatment guidelines for otitis media: the need for breadth and flexibility. Pediatr Infect Dis J. 1995;14(5):429-35.

34. Cohen R, Levy C, Hentgen V, Boucherat M, de La Rocque F, d'Athis P, Bingen E. Relationship between clinical signs and symptoms and nasopharyngeal flora in acute otitis media. Clin Microbiol Infect. 2006;12(7): 679-8.

35. Cohen R, Bingen E, Levy C, Thollot F, Boucherat M, Derkx V, Varon E. Nasopharyngeal flora in children with acute otitis media before and after implementation of 7 valent pneumococcal conjugate vaccine in France. BMC Infect Dis. 2012;7:12-52. https://doi.org/10.1186/1471-2334-12-52.

36. Kadurugamuwa JL, Sin L, Albert E, Yu J, Francis K, DeBoer M, et al. Direct continuous method for monitoring biofilm infection in a mouse model. Infect Immun. 2003;71(2):882-90.

37. Soto SM, Smithson A, Horcajada JP, Martinez JA, Mensa JP, Vila J. Implication of biofilm formation in the persistence of urinary tract infection caused by uropathogenic Escherichia coli. Clin Microbiol Infect. 2016;12(10): 1034-6.

38. Niu C, Gilbert ES. Colorimetric method for identifying plant essential oil components that affect biofilm formation and structure. Appl Environ Microbiol. 2004;70(12):6951-6

39. Naves P, del Prado G, Huelves L, Gracia M, Ruiz V, Blanco J, et al. Measurement of biofilm formation by clinical isolates of Escherichia coli is method- dependent. J Appl Microbiol. 2008;105(2):585-90.

40. Camilli R, Pantosti A, Baldassarri L. Contribution of serotype and genetic background to biofilm formation by Streptococcus pneumoniae. Eur I Clin Microbiol Infect Dis. 2011;30(97-102):829-33.

41. Webster P, Wu S, Gomez G, Apicella M, Plaut AG, St Geme JW. Distribution of bacterial proteins in biofilms formed by non-typeable Haemophilus influenzae. J Histochem Cytochem. 2006;54(7):829-42 Epub 2006 Mar 20.

42. Weimer KED, Armbruster CE, Juneau RA, Hong W, Pang B, Swords WE. Coinfection with Haemophilus influenzae promotes pneumococcal biofilm formation during experimental otitis media and impedes the progression of pneumococcal disease. J Infect Dis. 2010;202:1068-75.

43. Tikhomirova A, Kidd SP. Haemophilus influenzae and Streptococcus pneumoniae: living together in a biofilm. Pathog Dis. 2013;69(2):114-26. https://doi.org/10.1111/2049-632X.12073.

44. Shouval DS, Greenberg D, Givon-Lavi N, Porat N, Dagan R. Site-specific disease potential of individual Streptococcus pneumoniae serotypes in pediatric invasive disease, acute otitis media and acute conjunctivitis. Pediatr Infect Dis J. 2006;25(7):602-7.

45. Moriyama S, Hotomi M, Shimada J, Billal DS, Fujihara K, Yamanaka N Formation of biofilm by Haemophilus influenzae isolated from pediatric intractable otitis media. Auris Nasus Larynx. 2009:36(5):525-31.

46. Rayner MG, Zhang Y, Gorry MC, Chen Y, Post JC, Ehrlich GD. Evidence of bacterial metabolic activity in culture-negative otitis media with effusion. JAMA. 1998;279(4):296-9

47. Bingen $E$, Cohen R, Jourenkova N, Gehanno P. Epidemiologic study of conjunctivitis-otitis syndrome. Pediatr Infect Dis J. 2005;24(8):731-2.

48. van Dongen TM, van der Heijden GJ, van Zon A, Bogaert D, Sanders EA, Schilder AG. Evaluation of concordance between the microorganisms detected in the nasopharynx and middle ear of children with otitis media. Pediatr Infect Dis J. 2013:32(5):549-52.

Ready to submit your research? Choose BMC and benefit from:

- fast, convenient online submission

- thorough peer review by experienced researchers in your field

- rapid publication on acceptance

- support for research data, including large and complex data types

- gold Open Access which fosters wider collaboration and increased citations

- maximum visibility for your research: over $100 \mathrm{M}$ website views per year

At BMC, research is always in progress.

Learn more biomedcentral.com/submissions 